\title{
Do we need to extend CMV prophylaxis course in high risk patients post transplant? A single centre retrospective study of CMV infection in kidney transplant patients
}

\author{
M Ghazal Aswad, C Smith, S Khan, J Black, S Chughtai, S Dharmayan, A Ali, \\ P Rajagopal, T Doughman, A Bagul
}

Introduction: CMV infection is one of the most common viral infection post transplantation. It is associated with multiple morbidities including pneumonitis, colitis, retinitis, hepatitis, encephalitis and in severe form can potentially cause mortality. Hence majority of centres give CMV prophylaxis post transplantation in high risk recipient group (whose CMV status are negative and receive kidneys from CMV positive donors). However, the length of the prophylaxis course varies between three to six months among different transplant units. The aim of the study is to assess the incidence of CMV infection in our centre and evaluate the need to extend our current three month CMV prophylaxis course.

Methods: 95 patients who received kidney transplant between April 2015 and March 2016 were included. Donor and recipient CMV status was checked at the time of transplantation and patients received three month CMV prophylactic course of Valganciclovir if their CMV status was negative and they received a kidney from a CMV positive donor. Valganciclovir dose was titrated according to creatinine clearance and patients were followed up for a minimum of 6 months. CMV infection incidence was checked and the length of time to develop the infection was assessed.
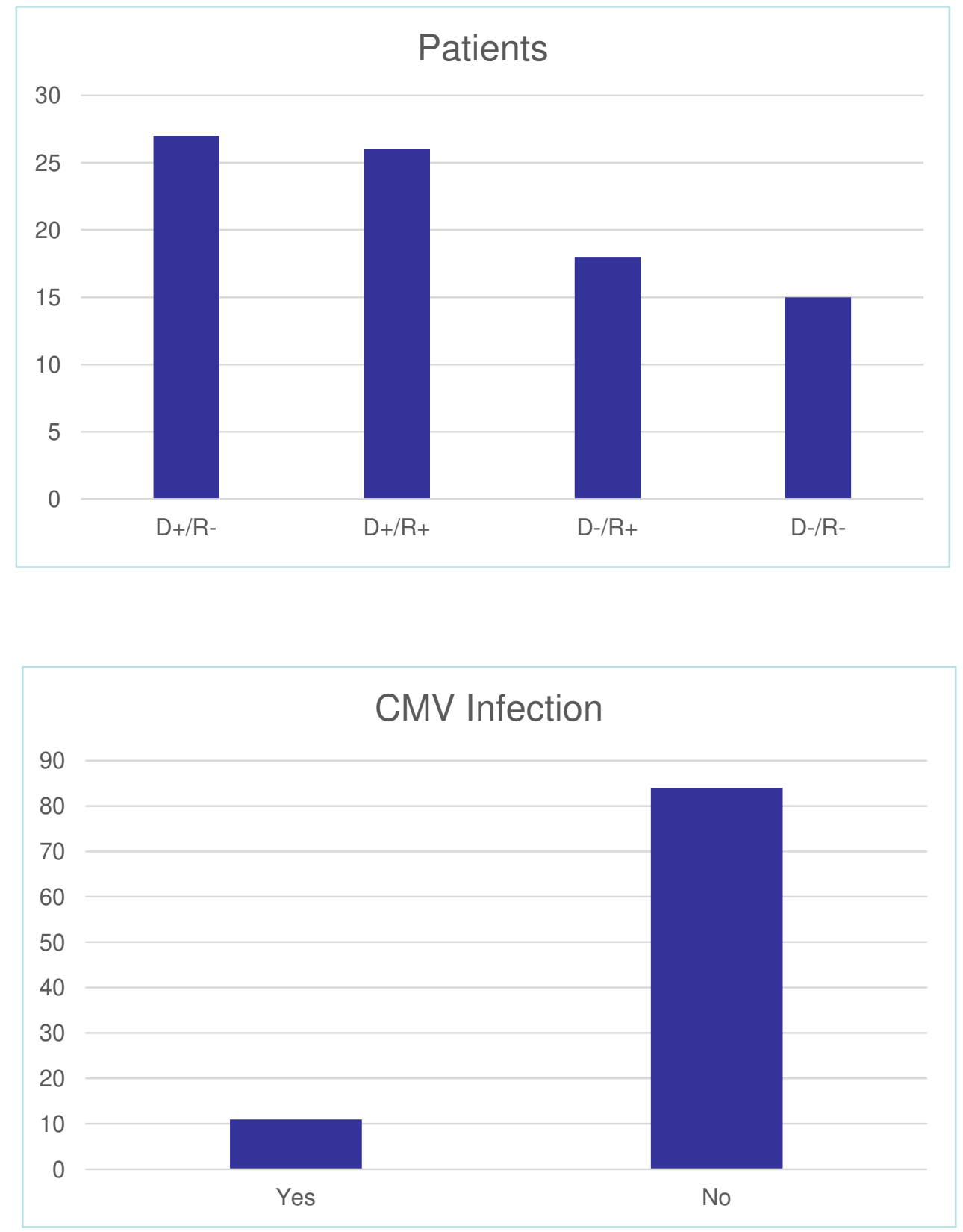

Results: 11 patients (11.6\%) developed CMV infection. From these patients, 9 (81.8\%) where form the high risk group and received CMV prophylaxis for 3 months whereas $2(18.1 \%)$ where from the low risk group and didn't receive prophylaxis post op as per unit protocol. 8 patients $(72.7 \%)$ developed the infection between four to six months post operatively and 3 patients (27.3\%) acquired the infection within three months. Mean creatinine level at 6 months for infected group and negative group was $175 \mu \mathrm{mol} / \mathrm{l}$ and $170 \mu \mathrm{mol} / /$ respectively.
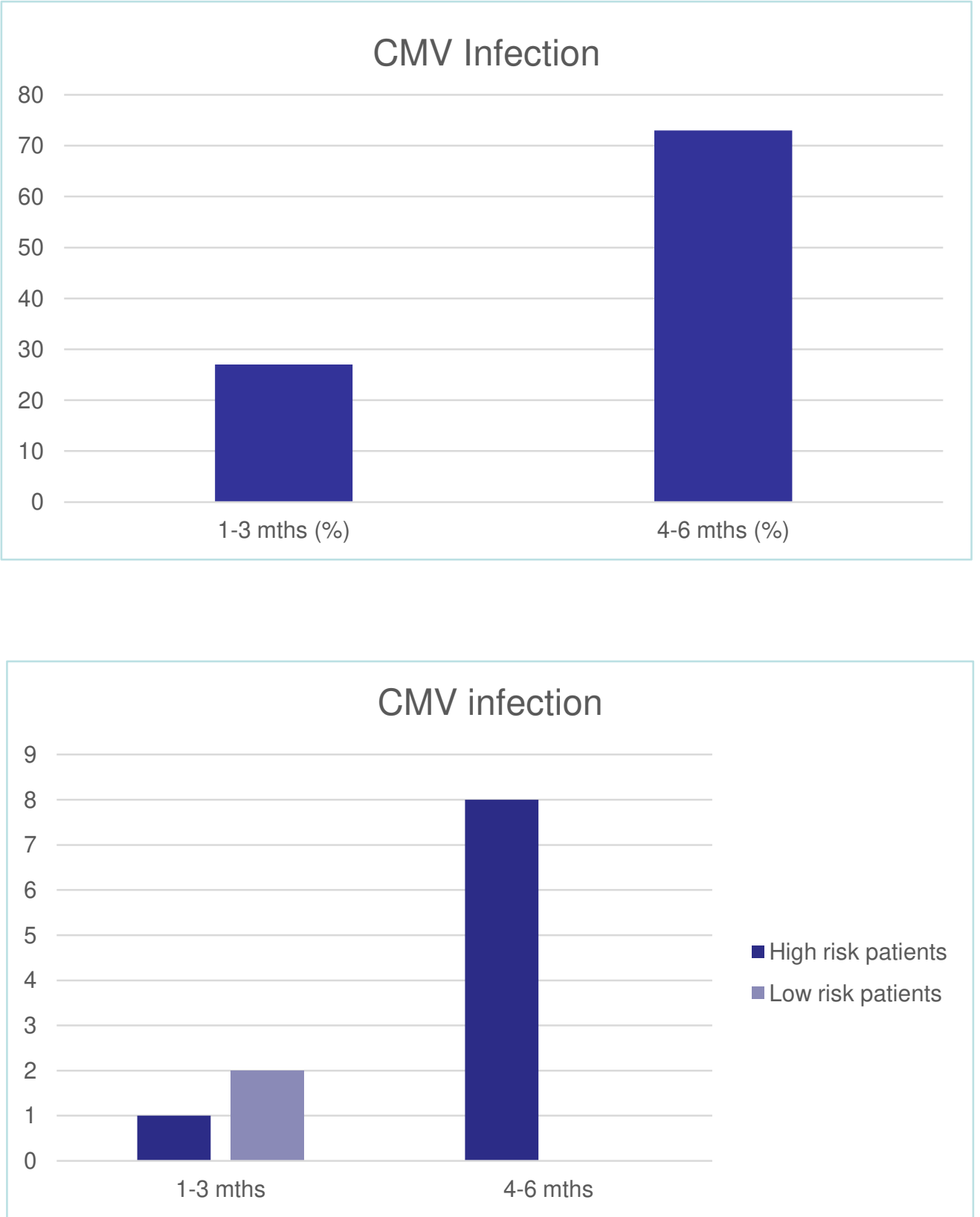

Conclusion: CMV infection is one of the major viral infections post transplant. In our unit around three quarters of the infections occurred between four to six months post operatively despite having CMV prophylaxis for the initial three months. We recommend from our study that the current three month CMV prophylaxis protocol in high risk patients is extended to six months in our unit and a repeat study with larger number of patients is conducted in the future to evaluate the incidence of CMV infection after implementation of the six months CMV prophylaxis protocol.

References:

Humar, A., Lebranchu, Y., Vincenti, F., Blumberg, E.A., Punch, J.D., Limaye, A.P., Abramowicz, D., Jardine, A.G., Voulgari, A.T., Ives, J., Hauser, I.A. and Peeters, P. (2010) 'The efficacy and safety of 200 days Valganciclovir Cytomegalovirus Prophylaxis in high-risk kidney transplant recipients', American Journal of Transplantation, 10(5), pp. 1228-1237. doi: 10.1111/j.1600-6143.2010.03074.x. 